\title{
Theorien und Modelle zur Entstehung von Aufmerksamkeit in der Wissenschaft
}

Nachdem der Ausgangspunkt und das Ziel dieser Arbeit dargelegt worden sind, werden in diesem Kapitel bestehende Ansätze der Wissenschaftssoziologie aufgearbeitet, die sich bereits ähnlichen Fragestellungen widmen. Zwar gibt es keine Arbeiten, die in vergleichbarer Weise nach der Entstehung von Aufmerksamkeit gegenüber Themen fragen oder die gar das Themenkarrieren-Konzept auf die Wissenschaft anwenden. Nach einer Ausweitung der Suche auf MakroBeschreibungen und -Erklärungen für das Aufkommen und Abklingen der fachgemeinschaftlichen Beschäftigung mit bestimmten Theorien, Methoden, Schulen oder wissenschaftlichen Modeerscheinungen geraten jedoch einige Theorien und Modelle in den Blick. Diese werden im Folgenden systematisiert und erläutert, sodass einerseits bereits vorhandene Erkenntnisse für die Beantwortung der Fragestellung erschlossen werden, andererseits aber auch die Forschungslücke sichtbar gemacht wird, deren Problematisierung und Schließung diese Arbeit anstrebt. Dementsprechend zielt dieses Kapitel darauf ab, zum einen die Deduktion zu beachtender Mechanismen für die Entwicklung der Forschungsheuristik zu ermöglichen (siehe Kp. 5) sowie zum anderen die Kontextualisierung der eigenen Erkenntnisse im Rahmen des aktuellen wissenschaftssoziologischen Theoriekanons zu gewährleisten (siehe Kp. 9 und 10).

Dieses Kapitel folgt also der Frage, welche Ansätze zur Erklärung der Entstehung von Aufmerksamkeit in der Wissenschaft bereits existieren und welche Erkenntnisse sich daraus für die Verfolgung der dieser Arbeit zugrunde liegenden Fragestellung ableiten lassen. Hierfür werden vier Theoriestränge voneinander abgegrenzt, erläutert und auf Schnittmengen mit der Fragestellung dieser Arbeit geprüft: Zunächst wird mit dem „Paradigmenwechsel“ nach Kuhn eine der bis heute einflussreichsten wissenschaftssoziologischen Theorien zur Erklärung wissenschaftlicher Transformationen dargelegt (Kp. 2.1). Eine wesentliche

A. Gravert, Themenkarrieren in der Wissenschaft, 
Grundlage Kuhns stellen Flecks „Denkkollektive“ dar, in deren Tradition Theorien zur Entstehung „Epistemischer Gemeinschaften“ zu sehen sind (Kp. 2.2). Mit ,theory groups“ und „invisible colleges“ werden in Abschnitt 2.3 zwei auf den Lehrstuhl von De Solla Price zurückgehende Theorien zur Diffusion wissenschaftlicher Schulen vorgestellt. Schließlich werden die normativen Diskussionen unterschiedlichster Fachrichtungen zu „Modeerscheinungen “ oder "fashions “ in der Wissenschaft gesammelt und in einen Zusammenhang gebracht (Kp. 2.4).

\subsection{Wissenschaftliche Revolutionen und Paradigmenwettstreit}

Thomas Kuhns „Struktur Wissenschaftlicher Revolutionen“ (1970 [1962]) war konstitutiv für die Entstehung der Wissenschaftssoziologie in ihrer jetzigen Form und ist bis heute das meistzitierte Buch der Sozialwissenschaften ${ }^{1}$. Die darin enthaltene revolutionäre These Kuhns richtet sich gegen die Auffassung, wissenschaftliche Entwicklung folge dem Muster des inkrementellen und kumulativen Fortschritts wie es Mertons Ethos und Poppers Falsifikationismus vorgaben. Vielmehr handele es sich um eine Abfolge „wissenschaftlicher Revolutionen “, also nicht-kumulativer Umbrïche in Form von Ablösungen eines alten durch ein neues Paradigma, die jeweils von Perioden ,normaler Wissenschaft “ (,normal science ") gefolgt seien (Kornmesser und Schurz 2014b: 12; Weingart 2003: 43).

Grundlegend für Kuhns Theorie wissenschaftlicher Revolutionen ist der Begriff des „Paradigmas“. Kuhn knüpft damit an Flecks (1935) Konzept der „Denkkollektive“ (siehe Kp. 2.2) an und argumentiert, Forscher*innen eines wissenschaftlichen Feldes würden in der Regel im Einklang mit einem Paradigma denken und handeln (Starbuck 2009: 109). Dieses Paradigma gibt der Fachgemeinschaft Modellprobleme und Modelllösungen vor (Kuhn 1970 [1962]: viii) und wird beschrieben als die ganze Konstellation von theoretischen Annahmen, Methoden, Meinungen, Werten, Relevanzkriterien usw., die von den Mitgliedern einer wissenschaftlichen Gemeinschaft geteilt werden (Lietz 2013: 360). Umgekehrt lässt sich eine „scientific community“ als eine Sammlung von Wissenschaftler*innen fassen, die ein gemeinsames Paradigma teilen (Kuhn 1970 [1962]: 176). Diese zirkuläre Bestimmung und die erkennbare Unschärfe des Paradigma-Begriffs ist Kernpunkt der Kritik an Kuhns Theorie und wird später

\footnotetext{
${ }^{1} \mathrm{Zu}$ dieser Aussage kommen van Noorden et al. (2014) sowie Green (2016) auf der Basis von Google Schoolar Daten. Eine Abfrage am 29. Januar 2020 kommt zu dem gleichen Ergebnis. Bestimmte methodologische Bücher (bspw. Yin 2009) vereinen aber mehr Zitationen, wenn alle überarbeiteten Auflagen zusammengezählt werden.
} 
auch durch den Urheber selbst eingeräumt (vgl. Musgrave und Lakatos 1970; Lietz 2013: 360; Kornmesser und Schurz 2014b: 17).

Paradigmen konstituieren Perioden kumulativer Wissensproduktion. In diesen Phasen ,normaler' Wissenschaft betreiben Wissenschaftler*innen ,puzzle solving ", das heißt, sie forschen im Einklang mit dem vorherrschenden Paradigma, ohne dieses in Frage zu stellen, und unternehmen so den Versuch „to force nature into the preformed and relatively inflexible box that the paradigm supplies " (Kuhn 1970 [1962]: 24). Mit der Zeit häufen sich jedoch unerwartete Phänomene (,,anomalies"), die nicht mit dem vorherrschenden Paradigma vereinbar sind. Ist eine kritische Masse solcher Anomalien erreicht, wird die Geltung des Paradigmas zunehmend in Zweifel gezogen und es gerät in eine Krise (Bettencourt et al. 2009: 212). Der Paradigmenwechsel ist dann das relativ abrupte Ereignis, in dem ein neues Paradigma aus zunächst wettstreitenden Interpretationen der Anomalien entsteht und an Zuspruch gewinnt, sodass der Zyklus von Neuem mit einer Periode ,normaler' Wissenschaft beginnen kann (Lietz 2013: 359). Für die Auswahl des neuen Paradigmas durch die Fachgemeinschaft können verschiedene Faktoren (gleichzeitig) eine Rolle spielen, darunter auch ästhetische oder psychologische.

Häufig wird Kuhns Aussage hervorgehoben, das alte und das neue Paradigma seien ,,inkommensurabel“, das heißt, die jeweiligen Theorien, Problemstellungen und Konzepte seien nicht vereinbar und ,nicht durch rationale Argumente aufeinander beziehbar" (Weingart 2003: 43). Allerdings stellt Kuhn später gegenüber dieser radikalen Lesart klar, dass unterschiedliche Paradigmen sehr wohl „lokale " bzw. begrenzte Kommensurabilitäten aufweisen können (Kornmesser und Schurz 2014b: 25-29). Aufgrund der Radikalität der Umstellung und der Persistenz etablierter Machtstrukturen in der wissenschaftlichen Gemeinschaft bewirkt in der Regel erst ein Generationswechsel die Ablösung des alten Paradigmas durch ein neues (Weingart 2003: 43). Kuhn führt eine Reihe wissenschaftlicher Revolutionen als Beispiele für seine Theorie an, darunter jene, die mit den Namen Kopernikus, Newton oder Einstein in Zusammenhang stehen (Kuhn 1970 [1962]: 6). Allerdings weist er darauf hin, dass Paradigmenwechsel nicht notwendigerweise dieses Ausmaß annehmen müssen, sondern dass auch einzelne Entdeckungen und kleine Gruppen von ca. 25 Personen revolutionär sein können (Lietz 2013: 359).

Im Rahmen eines Paradigmenwechsels ändern sich die Kriterien, die die Legitimität von Problemen und Lösungen determinieren (Kuhn 1970 [1962]: 109). Folglich ändert sich nicht nur der Blickwinkel, sondern auch der Inhalt der Forschung: 
„Led by a new paradigm, scientists adopt new instruments and look in new places. Even more important, during revolutions scientists see new and different things when looking with familiar instruments in places they have looked before. " (Kuhn 1970 [1962]: 111)

Hier weicht Kuhns Theorie in einem weiteren (vgl. Kp. 1.3.1), für diese Arbeit entscheidenden, Punkt von Merton und Popper ab: Im Gegensatz zu der bei Merton und Popper vorherrschenden Vorstellung, alle Individuen handelten nach universalen Regeln der Wissenschaft, rückt Kuhn die Paradigmengruppen als handlungsleitend in den Fokus, wie Weingart (2003: 43-44) ausführt:

\begin{abstract}
„Das jeweilige Paradigma hat einen analogen Status zu dem der Normen, d. h., es ist handlungsleitend für die ihm verpflichteten Wissenschaftler bzw. präziser für die betreffende Kommunikationsgemeinschaft oder scientific community. Das einer bestimmten Gruppe eigene Paradigma orientiert ihr Handeln, es strukturiert den Erkenntnisprozess, bestimmt die Probleme und legitimiert deren Lösungen. In diesem Bezug auf die scientific community sieht Kuhn die, soziologische Basis'seiner Position (vgl. Kuhn 1970: 252). Aufgrund dessen sind scientific communities für Kuhn und die an ihn anschließenden Wissenschaftssoziologen gleichbedeutend mit den, $\mathrm{Pa}$ radigmengruppen'; sie sind sowohl Argumentations- (bzw. Kommunikations-) als auch Handlungszusammenhänge. “
\end{abstract}

Die unterschiedlichen Positionen lassen sich anhand der Krise veranschaulichen, in der Wissenschaftler*innen sich für oder gegen eine neue Theorie entscheiden müssen. Im kritischen Rationalismus würden Individuen diese Entscheidung auf der Grundlage wohldefinierter Regeln fällen, mit dem Ziel, der Wahrheit näher zu kommen. Kuhn dagegen vertritt die Ansicht, dass neben individuellen Faktoren wie wissenschaftlicher Erfahrung oder Risikobereitschaft vor allem die durch die Paradigmengruppe vermittelten Handlungsorientierungen und epistemischen Werte diese Entscheidung anleiten (Hoyningen-Huene und Lohse 2012: 78-79). Gemäß Weingart (2003: 44) öffnet Kuhns Theorie damit „der Wissenschaftssoziologie die Tür zur soziologischen Analyse wissenschaftlicher Inhalte und deren Verknüpfung mit der wissenschaftlichen Sozialstruktur", das heißt zu einer „soziologischen Theorie wissenschaftlicher Entwicklung “.

Kuhns Theorie der Paradigmenwechsel hat bis heute großen Einfluss auf die Wissenschaftssoziologie, gleichzeitig hat sie aber auch anhaltende Kritik auf sich gezogen. Die seit der Buchveröffentlichung im Jahr 1962 andauernde Kuhn-Kontroverse lässt sich so zusammenfassen, dass die oben geschilderten, grundlegenden wissenschaftstheoretischen Einsichten Kuhns - bspw. in Bezug auf das Konzept der Inkommensurabilität - von den Übertreibungen einer radikalen Lesart gelöst und in gemäßigter und sich wandelnder Form in den aktuellen 
Kanon wissenschaftssoziologischer Theorien eingepasst werden (Schurz 2014: 48).

Kornmesser und Schurz (2014a) relativieren etwa die Vorstellung, wissenschaftliche Erkenntnisentstehung sei nur als diachrone Ablösung allgemeingültiger Paradigmen zu verstehen, die nicht miteinander vergleichbar seien (Peer und Sondermann 2016: 35). Stattdessen argumentieren sie, dass mehrere rivalisierende Paradigmen parallel existieren können und legen eine auf Kuhn aufbauende Forschungsheuristik zur Analyse dieser Multiparadigmatizität vor. Ihr aktualisierter Begriff des Paradigmas, zunächst als „kognitives System“ (Schurz 1998) eingeführt, ist auf die vergleichende Analyse koexistierender Paradigmen ausgerichtet. Dies impliziert, dass begrenzte Kommensurabilitäten nicht nur eingeräumt, sondern auch anhand der unterschiedlichen Elemente des Paradigmas analytisch gefasst werden. So besteht ein Paradigma aus vier Komponenten: einer theoretischen (Theoriekern einschl. Gesetzeshypothesen, Modellvorstellungen) einer empirischen (Musterbeispiele, exemplarische Anwendungsbereiche), einer methodologischen (erkenntnistheoretische Annahmen, Methodenpräferenzen, Relevanzkriterien) und einer programmatischen Komponente (Erkenntniserwartungen, gemeinschaftliches Ziel) (Kornmesser und Schurz 2014b: 16-25). Zu den unterschiedlichen Varianten der Koexistenz von Paradigmen und der sie vertretenden wissenschaftlichen Gemeinschaften zählt bspw. „Ignoranz und Eklektizismus“. Hierzu gehören zum einen das gegenseitige Ausblenden des jeweils anderen Paradigmas sowie zum anderen - im Falle einer gemeinsamen Betrachtung beider Paradigmen - die beliebige Vermischung unter Nichtberücksichtigung methodischer und theoretischer Konfliktlinien. Zwei weitere Varianten sind die „destruktive Rivalität“, also die einem fruchtbaren Austausch im Wege stehende ideologische Auseinandersetzung rivalisierender Paradigmengruppen sowie die „,konstruktive Rivalität“, bei der im Rahmen eines fruchtbaren Austauschs Positionen des rivalisierenden Paradigmas in das eigene eingebunden werden (ebd.: 34-36).

Die empirische Anwendung dieser Heuristik führt Schurz (2014: 57-59) zu der Diagnose, dass Formen des konstruktiven Austauschs zwischen zwei Paradigmen mit ähnlichem Gegenstandsbereich selten zu beobachten sind und dass stattdessen ideologische Kämpfe oder ein ,ignoranter Pluralismus “ vorherrschen. Diesen Umstand führt er auf die gegenwärtige Organisationsform der Wissenschaft zurück, in der über Karrierechancen innerhalb der hochspezialisierten wissenschaftlichen Gemeinschaften entschieden wird. Um diese Karrierechancen erhalten zu können, müssen sich Nachwuchswissenschaftler*innen spezialisieren 
und sich einer dieser Communities zurechnen. Die Kombination zweier rivalisierender Fachgebiete wird dagegen, zumindest seitens der Paradigmengruppen, nicht belohnt und teilweise sogar sanktioniert (Schurz 2014: 57-59).

\section{Schlussfolgerungen}

Kuhns Theorie verknüpft die epistemische Entwicklung einer Disziplin mit den Mechanismen ihrer Sozialstruktur. Bezogen auf ein Thema ist es demnach nicht eine-wie auch immer zu bestimmende - objektive Wichtigkeit, die dessen Erhebung zum präferierten Forschungsgegenstand einer Fachgemeinschaft bedingt. Stattdessen sind es soziale Faktoren, die dazu führen, dass wissenschaftliche Akteur*innen ein Thema vorantreiben oder blockieren. Hierzu zählen insbesondere ästhetische Präferenzen, strategische Interessen, die soziale Nähe von Wissenschaftler*innen sowie Generationenkonflikte. Oft sind es etablierte Akteur*innen, die den Status Quo verteidigen, während aufstrebende Wissenschaftler*innen in besonderem Maße für die Modifikation bestehender Deutungen, Relevanzen und Wissensstrukturen eintreten. Mit der Aktualisierung durch Kornmesser und Schurz wird Kuhns Theorie für die Analyse synchroner, multiparadigmatischer Strukturen angepasst. Diese Modifizierung scheint auch für die Betrachtung der Planungswissenschaft die passfähigere Variante, da hier bislang keine wissenschaftliche Revolution zu verzeichnen war, in der ein Paradigma vollständig zugunsten eines anderen aufgegeben wurde, wohl aber verschiedene Akteur*innengruppen beobachtet werden können, die zeitgleich unterschiedlichen Ansätzen in Bezug auf die theoretische, die methodologische sowie auf die programmatische Komponente folgen. Der nicht zu übersehende Nachteil dieser Aktualisierung von Kuhns Theorie ist allerdings, dass der Erklärungsgehalt in Bezug auf wissenschaftliche Transformationen verloren geht. Stattdessen werden anhand zahlreicher empirischer Beispiele die Autopoiesis und die Persistenz von Paradigmengruppen vor Augen geführt. Wie angesichts multiparadigmatischer Strukturen dennoch zu Erklärungsansätzen für wissenschaftliche Transformationen gelangt werden kann, zeigen die folgenden Teilkapitel (2.2, 2.3 und 2.4).

\subsection{Denkkollektive und Epistemische Gemeinschaften}

Das oben ausgeführte Verständnis Kuhns, nachdem Inhalte sowie Art und Weise des wissenschaftlichen Erkenntnisfortschritts unmittelbar mit der sozialen Struktur zusammenhängen, ist maßgeblich durch Ludwik Flecks Buch „Entstehung und 
Entwicklung einer wissenschaftlichen Tatsache“ (1935) beeinflusst, das vor dessen Wiederentdeckung durch Kuhn jahrzehntelang unbeachtet blieb (Kuhn 1970 [1962]: vi-vii; Egloff 2005: 7). Fleck (1935: 46) erläutert darin seine Idee der Denkkollektive, die er ,als Gemeinschaft der Menschen, die im Gedankenaustausch oder in gedanklicher Wechselwirkung stehen ", umreißt. Im Kontrast zu der damals vorherrschenden, individualistischen Erkenntnistheorie, ist für Fleck dieses Kollektiv Ausgangspunkt für Erkenntnis (Grimm 2012: 39). Analog zu den später von Kuhn aufgebrachten wissenschaftlichen Gemeinschaften als Trägerinnen von Paradigmen, sind Denkkollektive ,Träger geschichtlicher Entwicklung eines Denkgebietes, eines bestimmten Wissensbestandes und Kulturstandes, also eines besonderen Denkstiles “ (Fleck 1935: 46). Der Denkstil wird als ein geteilter kognitiver Rahmen beschrieben, der gemeinsame Perspektiven, Methoden, Techniken, Standards und literarische Stile umfasst. Er besteht aus einer bestimmten „Stimmung“, die die Bereitschaft für selektives Wahrnehmen und entsprechend gerichtetes Handeln umfasst (Hackett et al. 2017: 740; Fleck 1935: 105-106). Je nach Denkstil können Wissen und Wahrheit unterschiedlich ausfallen (Grimm 2012: 40). Diese Kontextabhängigkeit und Veränderlichkeit wissenschaftlicher Erkenntnis ist für Fleck eine Selbstverständlichkeit:

„Unmöglich ist ein wirklich isolierter Forscher, unmöglich ist eine ahistorische Entdeckung, unmöglich ist eine stillose Beobachtung. Ein isolierter Forscher ohne Vorurteile und ohne Tradition, ohne auf ihn wirkende Kräfte einer Denkgesellschaft und ohne Einfluß der Evolution wäre blind und gedankenlos. Das Denken ist eine kollektive Tätigkeit wie der Chorgesang und das Gespräch. Es unterliegt spezifischen Veränderungen mit der Zeit; es zeigt die geschichtliche Kontinuität dieser Veränderungen auf. [...] Was wir denken und wie wir sehen, hängt vom Denkkollektiv ab, dem wir angehören. “(Fleck 1983b [1935]: 81-82)

Miller und Fox greifen dieses Argument in ihrer Interpretation der ,epistemic community“ auf, die sie, in Anlehnung an Kuhns Paradigmengruppen, als „group of inquirers who have knowledge problems to solve" definieren (Miller und Fox 2001: 669). Diese strukturieren die Erkenntnisgewinnung ihrer Mitglieder durch tradierte Institutionen sowie durch aktuelle Forschungsaktivitäten und definieren, was möglich und was relevant ist.

Das Konzept der Denkkollektive ist nicht allein auf wissenschaftliche Gemeinschaften anwendbar, sondern auf alle Arten sozialer Gruppierungen. Damit einhergehend gehört ein moderner Mensch stets mehreren Denkkollektiven an, etwa im Rahmen eines Berufs, einer Religion oder einer Partei (Fleck 1983a [1936]: 114). Nicht zuletzt diese fortwährende Interaktion zwischen esoterischen 
(spezialisierte Wissenschaftler*innen) und exoterischen (gebildete Laien) Denkkollektiven stimuliert wissenschaftliche Entwicklung. In dieser engen Verknüpfung zwischen Wissenschaft und Gesellschaft liegt ein wesentlicher Unterschied zu Kuhn, der Wissenschaft grundsätzlich als losgelöst von anderen gesellschaftlichen Bereichen betrachtet und exogene Einflüsse auf Paradigmenwechsel zwar einräumt, aber nicht konzeptualisiert (Kuhn 1970 [1962]: x; Egloff 2005: 7). Flecks Ansatz eignet sich daher nicht nur für die Analyse von Forschungskollektiven und wissenschaftlichen Disziplinen, ,, er leistet auch gute Dienste für das Verstehen von interdisziplinären bzw. transdisziplinären Prozessen sowie für die Untersuchung von Interaktionen zwischen akademischen und nichtakademischen Wissenskulturen ", so Egloff (2005: 7).

Die Ansicht, Wissen sei kontextabhängig und die disziplinübergreifende, soziale Gruppe stelle und teile diesen Kontext, wird 1968 von dem Soziologen Holzner (1968) in seinem Konzept der epistemic community weitergeführt. Holzner und Marx (1979: 108) definieren den Begriff später als „knowledgeoriented work communities in which cultural standards and social arrangements inter-penetrate around a primary commitment to epistemic criteria in knowledge production and application ". Neben dem Glauben an die wissenschaftliche Methode als Zugang zur Wahrheit eint die Epistemische Gemeinschaft die geteilte Wissensbasis, über die sie ein Selbstverständnis und eine Einordnung in die Umgebung entwickelt (Haas 1992: 3).

Bezug nehmend auf Kuhn und Holzner, bringt Haas den Begriff der Epistemischen Gemeinschaften Anfang der 1990er in der Politikwissenschaft, konkreter im Fachbereich Internationale Beziehungen, ein und führt ihn damit zu neuer Bekanntheit (Cross 2013: 141). Er definiert epistemic communities als ,network of professionals with recognized expertise and competence in a particular domain and an authoritative claim to policy-relevant knowledge within that domain or issue-area " (Haas 1992: 3). Bei Haas können Epistemische Gemeinschaften aus Expert*innen unterschiedlicher Disziplinen und Hintergründe bestehen und es muss keine direkte Interaktion zwischen den Mitgliedern stattfinden. Die Affiliation zur Gruppe ergibt sich stattdessen aus (1.) gemeinsamen normativen Überzeugungen, (2.) geteilten Ansichten zu kausalen Zusammenhängen bezüglich Problementstehung und Problemlösung, (3.) gemeinsamen Kriterien zur Validierung von Wissen in ihrer Domäne und (4.) einem gemeinsamen Politikziel (ebd.: 3). Das staatenübergreifende Teilen dieser durch wissenschaftliches Wissen geprägten Orientierungen ermöglicht gemäß Haas die internationale Kooperation im Hinblick auf globale Probleme, die das primäre Erkenntnisinteresse dieser Forschungstradition darstellt (Haas 1992: 4 ff.; Weingart 2003: 130). 
Auch wenn wissenschaftliches Wissen und die Beteiligung von Wissenschaftler*innen in Epistemischen Gemeinschaften in dem weitverbreiteten Verständnis von Haas eine zentrale Rolle spielen, wird die exklusive Anwendung des Konzepts auf die Wissenschaft mehr und mehr abgelöst (Meyer und MolyneuxHodgson 2010: Abschn. 1.2; Haas 1992: 3). Stattdessen wird insbesondere das Bestreben, politische Entscheidungsträger*innen durch die Vermittlung von Expert*innenwissen von den eigenen Inhalten zu überzeugen, als das konstituierende Element Epistemischer Gemeinschaften angesehen (Dunlop 2014, 2017). Durch die Analyse der in diesem Sinne mehr oder weniger erfolgreichen Kommunikationen und der dazugehörigen Rückkopplungseffekte zwischen wissensbasierten Gruppen und dem politischen System wird eine Verknüpfung politikwissenschaftlicher und wissen(schaft)ssoziologischer Ansätze geleistet. So legt Dunlop (2014: 216) die Risiken des „groupthink“ dar, die darin bestehen, dass bestimmte methodologische und normative Entscheidungen der Epistemischen Gemeinschaft zu einer Nichtberücksichtigung wichtiger Erkenntnisse rivalisierender Gruppen führen. Das Kämpfen um Politikrelevanz hat außerdem zur Folge, dass die Forschungskapazitäten auf den entscheidungsrelevanten Bereich fokussiert werden, sodass andere Forschungsbereiche vernachlässigt werden (Dunlop 2014: 209-216). Zudem resümiert Dunlop (2017: 215), dass insbesondere in komplexen Politikfragen und vor allem in der Agenda Setting-Phase des Politikprozesses, wenn die Wissensdefizite am größten sind, Epistemische Gemeinschaften Gehör in der Politik finden. Der Wissenstransfer in die Politik bleibt allerdings nicht ohne Risiko. So lässt sich bspw. kaum abschätzen in welcher Form die Politik das Wissen in Anspruch nimmt (Dunlop 2017).

Ein zentraler Kritikpunkt an der dargelegten Forschungstradition liegt darin, dass sie das wissenschaftlich fundierte Wissen der Epistemischen Gemeinschaft als anderen Wissensformen überlegen ansieht. Analog wird die Handlungsmotivation ihrer Mitglieder aus ihrer professionellen Expertise hergeleitet, während persönliche Interessen, strategische Motivationen und soziale Hintergründe ausgeblendet werden. Cross (2013: 159) hält dieses Verständnis für fehlgeleitet und weist darauf hin, dass die Anerkennung und Legitimierung von Expert*innenwissen sozial konstruiert ist. In einer Meta-Analyse fasst sie die Kontextbedingungen zusammen, die den Erfolg Epistemischer Gemeinschaften wahrscheinlicher machen. Demnach sind Epistemische Gemeinschaften besonders durchsetzungsstark, wenn 
„(Scope conditions)

- there is uncertainty surrounding the issue because it is complex or new (uncertainty from perceived crisis);

- the issue is surrounded by uncertainty and it is politically salient (continuous uncertainty);

- the decision-makers they are trying to persuade are unhappy with past policies and present problems (uncertainty from perceived crisis);

(Political opportunity structure)

- they have access to all necessary top decision-makers; they anticipate other actors' preferences and actions despite fluidity in the system [...];

(Phase in the policy process)

- they seek to influence the terms of the initial debate, instead of the decision itself;

- they deal with subsystem, technocratic phase of decisionmaking, rather than shaping broader political beliefs;

(Coalition building)

- the networks they are competing against are not as cohesive or certain of their aims; they share a high level of professional norms and status;

(Policy field coherence)

- there is respected quantitative data, instead of very subjective qualitative data." (Cross 2013: 144)

Die Überzeugungskraft Epistemischer Gemeinschaften ist dementsprechend weniger in den Inhalten bzw. deren Evidenz oder Validität zu suchen. Besonders überzeugend sind epistemic communities vielmehr dann, wenn deren Mitglieder über einen hohen Status verfügen, als integer gelten und sie auf der Basis eines weitreichenden, internen Konsenses agieren können und wollen (Cross 2013: 159). Cross betont weiterhin die Rolle gruppeninterner Mechanismen für den Erfolg Epistemischer Gemeinschaften. Diese interne Dynamik ist aber weniger durch die Art des Wissens konstituiert, sondern eher durch ihre interne Kohäsion und Professionalität (,professionalism“), also die Expertise, die geteilten Normen, die Arbeitskultur, das Engagement und die Intensität der Interaktion ihrer Mitglieder untereinander (ebd.: 149-151). 


\section{Schlussfolgerungen}

Das gegenüber klassisch wissenschaftssoziologischen Ansätzen hervorstechende Charakteristikum der Forschungstraditionen um Denkkollektive und Epistemische Gemeinschaften ist die analytische Aufhebung der Systemgrenzen zwischen Wissenschaft und Umwelt. Die Mitglieder der Gemeinschaften stammen aus unterschiedlichen Gesellschaftsbereichen und sie eint das Bestreben, wissensbasiert ihre Inhalte zu vermitteln und durchzusetzen. Diese Perspektive erscheint als fruchtbar für eine Anwendung im Feld der Raumplanung, denn hier sind die Grenzen zwischen Wissenschaft, Praxis, Politik und Gesellschaft mitunter durchlässig und veränderlich. Die Existenz Epistemischer Gemeinschaften aus Politiker*innen, Bürger*innen, Planer*innen und (Planungs-)Wissenschaftler*innen, die ein bestimmtes Thema durchsetzen oder blockieren wollen und dabei der Produktion und Vermittlung von Wissen eine große Rolle beimessen, scheint auch hier gegeben. Dies vorausgeschickt, erscheinen die oben dargelegten Faktoren und Mechanismen für eine weitere Betrachtung als besonders wertvoll. So kann festgehalten werden, dass für die strategische Durchsetzung der eigenen Interessen und für die Geltung des vorgelegten Wissens weniger die Art und Gesichertheit der zugrundeliegenden Evidenz ausschlaggebend sind, sondern vielmehr das konsensuale, koordinierte, professionelle und engagierte Agieren einer Gemeinschaft möglichst angesehener Akteur*innen. Außerdem ergeben sich aus den Kontextbedingungen Gelegenheitsfenster für die Verankerung eigener Inhalte, etwa wenn große Unsicherheit bezüglich des verhandelten Themas besteht und es auf der öffentlichen Agenda weit oben angesiedelt ist. Im Zuge ihres (teilweise wissenschaftsexternen) Aktivismus werden wichtige Erkenntnisse rivalisierender Gruppen ignoriert und eigene Forschungskapazitäten (insbesondere wissenschaftsintern) auf einen engen Bereich fokussiert, der für die Zielerreichung der Gruppe als wichtig eingeschätzt wird.

\subsection{Theory groups und invisible colleges: Die Entstehung und Diffusion wissenschaftlicher Felder}

Aufbauend auf Kuhns Paradigmenwechsel gehen Wissenschaftsforscher*innen seit den 1960er Jahren vermehrt der Frage nach, wie Fachbereiche (, specialties “) entstehen und sich etablieren. Dabei stellen sie fest, dass bahnbrechende konzeptuelle Veränderungen in erster Linie durch das strategische Handeln von Gruppen durchgesetzt und etabliert werden. Sie erklären daher - ähnlich wie die Theorien Epistemischer Gemeinschaften, aber unter Ausblendung systemexterner Faktoren - den persönlichen Kontakt und das gemeinsame strategische Programm von 
Gruppen zu den wesentlichen Voraussetzungen für Veränderungen in der Sozialund Wissensstruktur einer Disziplin. Dieser Standpunkt ist gemäß Weingart (2003: 47) darauf zurückzuführen, dass es ,,in den Umbruchphasen wissenschaftlicher Entwicklung keine rein rationalen Beweise für neue Wissensbehauptungen gibt, sondern neue Forschungsprogramme formuliert werden, deren weitreichende theoretische Implikationen erst durch längere Forschungsprozesse ausgelotet werden müssen ". Die Gruppen schaffen deshalb Sicherheit für ihre Mitglieder, indem sie nach innen die Verpflichtung auf die gemeinsame Wissensbehauptung gewährleisten und nach außen strategisch auf ihre Umwelt einwirken.

Ausgehend von Derek Price (1963, 1965), der eine strukturalistische Sicht auf die Entwicklung wissenschaftlicher Denkschulen einnimmt, werden quantitative Studien auf Basis von Daten zu Publikationen, Zitationen und KoAutorenschaften als Ausgangspunkt genommen, um Rückschlüsse auf die soziale Struktur wissenschaftlicher Gemeinschaften zu gewinnen (Mullins 1983: 320; Price 1963). Die empirischen Analysen stimmen darin überein, dass das Wachstum neuer Forschungsbereiche in der Form einer logistischen Funktion (bzw. S-Kurve) verläuft. So resümiert Bettencourt et al. (2008: 496), „many fields began with a burst of exponential growth followed by saturation and an eventual steady-state“. Price selbst entwickelt dabei das Konzept der „Unsichtbaren Kollegien " (,,invisible colleges"), also informeller Netzwerke mehr oder weniger kohäsiver Forschungsgruppen, die ein bestimmtes Paradigma teilen oder einem spezifischem Forschungsbereich angehören (Price 1963: 56 ff.; Leifeld 2009: 94). Ebenso weist Price anhand von Zitationsdaten die Existenz von "research fronts “ nach, also kleiner Mengen aktueller wissenschaftlicher Publikationen und Wissenschaftler*innen einer Disziplin, die sich durch starke gegenseitige Zitierung von dem übrigen Feld abheben (Lietz 2013: 357; Price 1965).

Mullins Konzept der ,theory groups“ (1973) baut auf den genannten Arbeiten von Price und Kuhn auf und erweitert diese um Ansätze des Soziologen Harrison White, der insbesondere die Bedeutung von Netzwerken herausstellt (White 1970; Mullins 1983: 320). Im Gegensatz zu Kuhn sieht Mullins wissenschaftliche Entwicklung nicht als eine Abfolge von Paradigmen, sondern als die Konkurrenz zwischen Denkschulen, also relativ dichten Netzwerken von Wissenschaftler*innen, ,die im engen Austausch miteinander eigene Denkansätze hervorbringen und sich in diesen bestärken " (Schmitt und Fuhse 2015: 16). Mullins beschreibt ein vierstufiges Phasenmodell der Entstehung einer Subdisziplin (,specialty“) (Mullins 1983; Griffith und Mullins 1972): In der ersten Phase (,normal stage“) produziert eine kleine, informelle Gruppe um einen ,intellectual leader" neue Erkenntnisse. Sie ist noch isoliert in ihrem Feld, in dem bislang andere Probleme, Ideen und Sichtweisen vorherrschen. In der zweiten Phase 
(,,network stage“) entwickelt sich ein größeres Netzwerk, wobei die meisten Mitglieder anfangs an derselben Forschungseinrichtung angebunden sind und von dort diffundieren. Intellektueller Erfolg und die Deklaration eines gemeinsamen wissenschaftlichen Programms distinguiert die Gruppe jetzt von der sie umgebenden Fachgemeinschaft. In der dritten Phase (,cluster stage“) kolonisieren die Mitglieder der Gruppe andere Institute:
„Organizational concerns for jobs, publications, and meetings, the organization of new research centers, and the continued recruitment of new members all become important. The group's publications begin to include secondary works that explain ideas, summarize developments, and so forth. "(Mullins 1983: 321)

In der vierten Phase (,specialty stage“) wird das Programm Routine und neue Ideen entstehen auch außerhalb der kolonisierten Institute. Die Gruppe und ihre Erkenntnisse werden nun selbst zum Kontext ,normaler' Wissenschaft, aus dem wiederum neue Theoriegruppen entstehen können. Gemäß Mullins (1983: 321) liegt dieser Prozess allen intellektuellen Netzwerken, die ein gemeinsames Paradigma unterstützen, zugrunde. Der Prozess dauert zwischen acht und fünfundzwanzig Jahren, wobei nicht alle Gruppen erfolgreich alle Phasen durchwandern. Einige scheitern aus internen Gründen, wie bspw. durch schlechte Führung oder unlösbare methodologische Probleme, andere aus externen Gründen, wie bspw. durch konkurrierende Gruppen oder soziale Umbrïche.

Zeitgleich mit Mullins entwickelte Crane (1972) das Konzept der Unsichtbaren Kollegien weiter, wobei sie die Theorie der Diffusion von Innovationen nach Rogers (1983 [1962]) auf neue, besonders stark wachsende Forschungsbereiche in der Soziologie und der Mathematik anwendet. Gemäß Crane (1972: 76) lässt sich die Wissenschaft betrachten als „an enormous cluster of innovations, of which the most successful are diffused by means of a logistic curve in all facets of scientific activity". Wie auch bei Innovationen außerhalb der Wissenschaft, diffundieren neue Forschungsbereiche primär durch persönlichen Kontakt, durch den ein stetig wachsendes Netzwerk von Anwender*innen (,,adopters“) entsteht. In diesem Netzwerk nimmt eine Teilgruppe besonders produktiver und zentraler Wissenschaftler*innen, das Unsichtbare Kollegium, die zentralen Knotenpositionen ein. Diese Elite ist geografisch voneinander getrennt, aber durch überwiegend schwache Verbindungen (Granovetter 1973) miteinander verknüpft, in diesem Fall also durch lose, für Außenstehende nicht unbedingt sichtbare Kontakte, über die sich die Wissenschaftler*innen informell bezüglich aktueller Entwicklungen und work in progress austauschen (Leifeld 2009: 94; Price 1986: 75; Zuccala 2004: 22). Um die lose verbundenen, zentralen Wissenschaftler*innen ist jeweils ein 
enges Netzwerk weniger produktiver „Kollaborateure“ gruppiert. Nachwuchswissenschaftler*innen werden in diesen Gruppen rekrutiert und sozialisiert, sie übernehmen also Forschungsinhalte und werden Teil eines solidarischen Netzwerks. So entsteht eine ,informelle Meso-Struktur“ (Leifeld 2009: 94) innerhalb des Forschungsfeldes, bei der separate, jeweils eng vernetzte Gruppen über informelle Beziehungen der führenden Wissenschaftler*innen verknüpft sind (Crane 1972: 54).

Durch das starke Wachstum des Forschungsbereichs, also durch die steigende Zahl von Wissenschaftler*innen und Publikationen, wird es zunehmend schwierig, das Feld zu überblicken. Der persönliche, informelle Austausch zentraler Akteur*innen verliert an Bedeutung und das Feld fragmentiert in Subgruppierungen bzw. Schulen. Dies wirkt sich wiederum negativ auf den Wissensaustausch zwischen den Gruppen aus, sodass neue Ideen nicht mehr im gesamten Feld anerkannt und aufgegriffen werden (Crane 1972: 77-78). Zudem erschöpfen sich die ursprünglichen Ideen und die Innovationsfähigkeit nimmt ab. Der Fachbereich wird sukzessive unattraktiver und ist weniger in der Lage, um neue Köpfe zu konkurrieren. Die wissenschaftliche Gemeinschaft wendet sich neuen, zukunftsträchtigeren Konzepten zu (ebd.: 84).

Crane betont die Bedeutung zentraler Akteur*innen und Publikationen im Diffusionsprozess (Crane 1972: 72 ff.). Sie führt dies anhand des Konzepts von „core and scatter" aus, also der wechselseitigen Abhängigkeit von einigen stark sichtbaren (core) und vielen weniger sichtbaren Wissenschaftler*innen (scatter). So gibt die Präsenz von Wissenschaftler*innen, die kontinuierlich und mit ausreichender Sichtbarkeit in einem Forschungsfeld arbeiten, Wissenschaftler*innen, die neu in das Feld eintreten, Orientierung und ermöglicht die Entstehung eines sozialen Zirkels. Zudem gewährleistet der Kern, dass wichtige Erkenntnisse nicht verloren gehen, sondern akkumuliert und verbreitet werden können (Crane 1969b: 348-349):

„If all the literature on a particular subject was so scattered, it would be impossible for scientists to build on each other's work. Each scientist would be working by himself and with perhaps a few other scientists whom he accidently discovered. The existence of a, core 'of journals in the literature, and of scientists in the problem area, provides a kind of repetitiveness in scientific communication, insuring that certain ideas will be repeated sufficiently so that the relevant scientists may be assured of receiving at least some of the currently important findings. " (Crane 1969b: 350)

Zentrale Wissenschaftler*innen in dem Feld filtern und bündeln also Forschungsergebnisse, sodass diese aus der Masse an wissenschaftlichem Output 
hervorstechen und nicht verloren gehen. Diejenigen Ergebnisse, die von den „produktiven ", besonders sichtbaren Wissenschaftler*innen aufgegriffen werden, sind dementsprechend auch diejenigen, die von dem gesamten Forschungsbereich verwendet werden (Crane 1972: 83). Angesichts dieser kohäsiven Funktion sind die zentralen Wissenschaftler*innen in der Lage, die Themenwahl und die Definition von Standards in einem Feld stark zu beeinflussen (Besio 2010: 121-122). Auch wenn einige Innovationen dabei nicht von den besonders produktiven Wissenschaftler*innen selbst hervorgebracht, sondern nur übermittelt werden, werden sie ihnen häufig fälschlicherweise zugeschrieben (Crane 1972: 74). Während der Kern also eine integrierende Funktion einnimmt, wirkt scatter - also für das Feld weniger zentrale Wissenschaftler*innen - desintegrierend, indem es Zugänge zu neuem Wissen schafft und damit verhindert, dass das Feld zu einem ,completely subjective, sect-like phenomenon" wird (Crane 1969b: 350).

Die Bedeutung produktiver Wissenschaftler*innen - also solcher mit einer hohen Publikationsfrequenz - nimmt während der Wachstumsphase des Forschungsbereichs zu. Analog dazu nehmen relativ unproduktive Autor*innen nur Einfluss auf den Forschungsbereich, wenn sie zu Beginn des Diffusionsprozesses veröffentlichen und nicht zu einem späteren Zeitpunkt (Crane 1972: 75 ff.). Crane macht anhand des Diffusionsprozesses verschiedene Funktionen von Publikationen fest und wendet sich damit gegen die Interpretation, eine hohe Zitationsfrequenz sei ein Maß für die Qualität einer Publikation, wie sie etwa Cole (1970) vertritt. So sei etwa die erste Generation von Veröffentlichungen in einem neuen Forschungsbereich geprägt durch eine breit angelegte Literaturrecherche, während Veröffentlichungen der zweiten Generation eine stärkere Fokussierung innerhalb des aufgemachten Themenfelds vornehmen (Crane 1972: 76-77). Analog ließe sich argumentieren, Ideen der ersten Generation seien breiter angelegt, während diejenigen der zweiten Generation spezialisiert seien. Wäre dies der Fall, wäre die häufigere Zitierung - oder zumindest die Zitierung durch ein breiteres Spektrum von Veröffentlichungen - der Publikationen der ersten Generation nicht überraschend:

„Later and less cited papers in an area are exploring the implications of earlier work, eliminating false leads, and providing evidence for hypotheses. [...] They may be read and absorbed by the working scientist, but they do not contain the sort of material that itself generates new studies. “(Crane 1972: 77)

Die Aussicht einer Publikation auf Zitationen hängt also auch von ihrer Funktion im wissenschaftlichen Erkenntnisprozess ab. So könnte auch erklärt werden, warum theoretische Studien häufiger zitiert werden als empirische. Ideen werden 
in theoretischen Studien entwickelt und in empirischen Studien getestet. Während die theoretischen Ideengeber*innen in den empirischen Studien häufig zitiert werden, ist dies umgekehrt kaum der Fall (Crane 1972: 77).

Die Konzepte von Price, Mullins und Crane werden in einer Vielzahl von Studien aufgegriffen und in vielen Details bestätigt (für einen Überblick siehe Morris und Van der Veer Martens 2008). Im Gegensatz zu Mullins und Crane, die einen Nukleus der Entstehung eines neuen wissenschaftlichen Feldes in den Mittelpunkt stellen, betonen Hackett et al. (2017: 739-740) die parallele Entstehung verschiedener Nuklei:

\begin{abstract}
„Initially, researchers at several locations begin exploring similar problems without knowledge of each other's efforts, and publication is widely dispersed across different disciplinary journals. Through such publications researchers gradually become aware of their common interests. Dense channels of formal and informal communication arise among researchers, and these networks thicken to become the coherent groups and invisible colleges of the nascent research area."
\end{abstract}

Indem sich vermehrt Gelegenheiten für wissenschaftliche Debatten und informellen Austausch bieten, entsteht sukzessive ein gemeinsamer Denkstil im Sinne Flecks, also ein Konsens über Probleme, Definitionen, Techniken und Erkenntnisse.

Eine für diese Arbeit interessante Vertiefung bietet Michaelson (1993), die belegt, dass für die Entscheidung von Wissenschaftler*innen, in eine wissenschaftliche Gruppe einzutreten, persönliche Kontakte zu jeder Zeit ein großer Einflussfaktor sind, während das Rezipieren veröffentlichter Artikel erst nach einer gewissen Etablierung der Gruppe eine Rolle bei dieser Entscheidung spielt (Morris und Van der Veer Martens 2008: 231). Bettencourt et al. (2006; 2008) haben für mehrere Forschungsbereiche nachgewiesen, dass sich deren Wachstum gut durch ein modifiziertes Diffusionsmodell beschreiben lässt und dass die Diffusion qualitative, messbare Veränderungen in der Sozialstruktur der wissenschaftlichen Gemeinschaft induziert (Bettencourt et al. 2009: 212). So forschen in der Entdeckungsphase eines Forschungsbereichs lediglich wenige kleine Gruppen getrennt voneinander. Diffundiert der Forschungsbereich, erhöht sich nicht nur die Zahl involvierter Wissenschaftler*innen, sondern es verdichtet sich auch das Kollaborationsnetzwerk, das heißt, die Zahl der Beziehungen (Kollaborationen) pro Knoten (Wissenschaftler*innen) steigt. Erreicht die Dichte einen bestimmten, fachbereichsspezifischen Grenzwert, bildet sich sprunghaft eine Gigantische Komponente heraus: Das heißt, durch das Zusammenwachsen mehrerer Teilbereiche des Netzwerks ist plötzlich ein Großteil aller Autor*innen des Forschungsbereichs zumindest indirekt miteinander verbunden (siehe Kp. 
6.3.3; Bettencourt et al. 2009: 214). Diese Veränderung der Sozialstruktur verstehen Bettencourt et al. als Etablierung eines Paradigmas. Sie identifizieren also eine Dynamik auf Bedeutungsebene anhand ihrer Manifestation im sozialen Netzwerk der Fachgemeinschaft (Lietz 2013: 358). Die Anwendung von Kuhns Paradigmenwechsel auf die Entwicklung der Kollaborationsstrukturen zeigt Abb. 2.1, in der Modifikationen der Netzwerkstruktur den unterschiedlichen Phasen bzw. Phasenübergängen nach Kuhn zugeordnet werden:

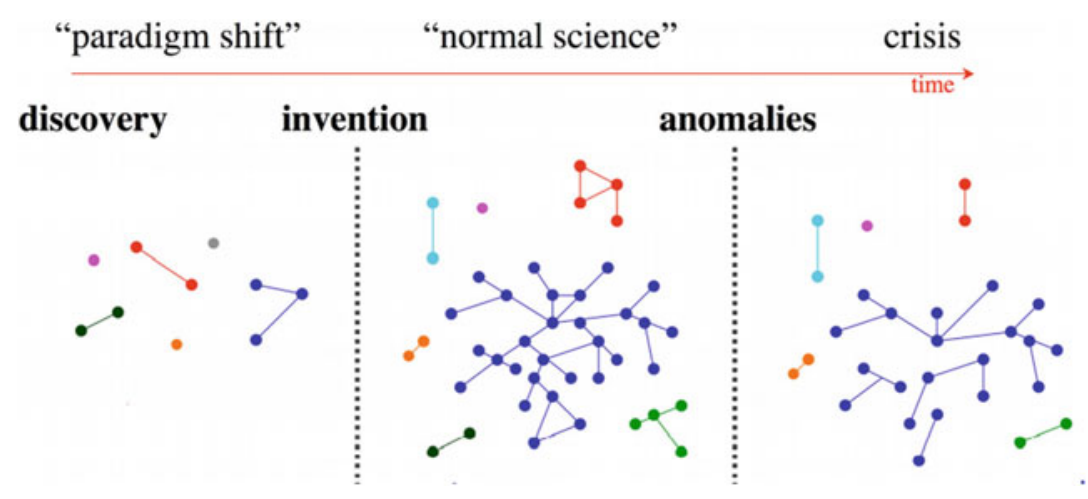

Abb. 2.1 Die Entstehung eines wissenschaftlichen Feldes (Quelle: Bettencourt et al. (2009: 213), Ausschnitt)

In Abb. 2.1 ist dargestellt, wie nach Bettencourt et al. neue wissenschaftliche Entdeckungen und deren Diffusion zu Veränderungen in der Struktur wissenschaftlicher Kollaborationen führen. Erfolgreiche Innovationen beginnen demnach typischerweise als kleine, unabhängige Aktivitäten (dargestellt als verschiedene, jeweils verbundene Komponenten unterschiedlicher Farbgebung). Diese wachsen zunächst getrennt voneinander, verbinden sich aber schließlich zu einer Gigantischen Komponente im Kollaborationsnetzwerk (dargestellt mittig in blau), die eine Phase ,normaler' Wissenschaft einläutet. Auch wenn die Größe und Wachstumsrate variieren, lässt sich diese Form der strukturellen Transformation - so die Hypothese - universell auf alle Fachbereiche anwenden (Bettencourt et al. 2009: 213). Die Innovation dieses Ansatzes liegt insbesondere darin, dass neue Forschungsbereiche mit Hilfe von Ko-Publikations- statt Zitationsanalysen (siehe Kp. 6.3.3) diagnostiziert werden und somit die soziale Ebene und die Bedeutungsebene konzeptionell miteinander verbunden werden. Gemäß Lietz 
(2013: 358) haben Bettencourt et al. damit „die Arbeit von Price mit den heutigen technischen Möglichkeiten aktualisiert und an Kuhns Theorie angebunden “.

\section{Schlussfolgerungen}

Die auf Price zurückgehenden Ansätze zur Entstehung von Fachbereichen bieten mehrere Anknüpfungspunkte für diese Arbeit. Zunächst heben sie den Zusammenhang von Veränderungen in der Wissensstruktur mit Modifikationen in der Sozialstruktur hervor und machen diesen Zusammenhang anhand von Zitationsund Ko-Publikationsnetzwerken sichtbar. Wissenschaftliche Transformationen entstehen demnach weniger durch das (alleinige) Rezipieren von Forschungsbeiträgen, sondern durch persönlichen Kontakt. Es entwickelt sich eine Sozialstruktur in der eine Elite untereinander lose in Verbindung steht und jeweils für sich eine Gruppe von Kollaborateur*innen um sich schart. Geht man - hier divergieren die Ansätze von zunächst getrennt voneinander wachsenden Komponenten bzw. Gruppen aus, so markiert der Zusammenschluss dieser Elite, also das Aufnehmen von Kollaborationsbeziehungen zwischen herausragenden Akteur*innen (,Makler*innen “, siehe 6.3.4) der jeweiligen Teilgruppen, den Phasenübergang von einer Entdeckungsphase in eine Phase ,normaler ' Wissenschaft. Durch die Verknüpfung der Teilgruppen entsteht eine Gigantische Komponente im Kollaborationsnetzwerk und der gemeinsame Wissensanspruch wird zur Routine. Indem die Innovation zum Kontext für die Entstehung neuer Innovationen wird, ist der Zerfall bzw. die Ausdifferenzierung der Wissens- und Sozialstruktur bereits in der Etablierung des Fachgebiets angelegt. Eine weitere im Rahmen dieser Arbeit zu berücksichtigende Erkenntnis liegt in der Rolle der Elite für die Kohäsion und Steuerung der fachgemeinschaftlichen Wissensund Sozialstruktur und in der Funktion (zunächst) nachgeordneter Forscher*innen für die Schaffung von Zugängen zu neuem Wissen. Ebenfalls fruchtbar erscheint die Differenzierung von grundlegenden Publikationen, die neue Ideen und Theorievorschläge aufwerfen, und den darauf folgenden Publikationen, die diese Innovationen prüfen. Letztere werden zwar deutlich weniger zitiert, dies lässt aber nicht unbedingt auf ihre geringere Bedeutung für die Disziplin schließen, sondern vielmehr auf ihre anders gelagerte Funktion.

\subsection{Modeerscheinungen in der Wissenschaft}

Modeerscheinungen in der Wissenschaft werden spätestens seit Anfang des 20. Jahrhunderts beobachtet (vgl. Sombart 1913: 140) und bis heute in unregelmäßigen Abständen in den verschiedensten Fachdisziplinen diskutiert (bspw. 
Soziologie, Sorokin 1956; Zell-Biologie, Fell 1960; Geomorphologie, Sherman 1996; Medizinische Soziologie, Barber 1968; Organisationstheorie, Bort und Kieser 2011). Den Ansätzen gemein ist der Versuch, gesellschaftliche oder sozialwissenschaftliche Konzeptionen von „Mode“, „fashion“, „fad“ oder „hype“ auf die Wissenschaft zu übertragen. In den Ansätzen ist jeweils ein Forschungsbereich, eine Theorie, ein methodischer Ansatz oder ein Paradigma - nicht ein Thema - der Gegenstand, der eine Mode erlebt. Dabei werden Moden in der Wissenschaft häufig in Opposition zu einer vermeintlich rationalen oder objektiven Wissenschaft gesehen und teilweise zur Falsifikation eines entsprechenden rationalistischen Wissenschaftsverständnisses angeführt.

Eine der wenigen, über szientometrische Kennziffern hinausgehenden, soziologischen Definitionen von Modeerscheinungen in der Wissenschaft stammt von Sperber (1990: 10-11):

„The fashion process is a form of collective behavior marked by a series of normative
preoccupations: keeping in step with the times, with the latest of developments; follo-
wing the examples of prestigious opinion leaders who , keep their ears to the ground
and articulate the shared and implicit sentiments of the public; admiring proposals for
adoption when they are in good taste and new, and discarding them when they are in
bad taste and old; dismissing the weight of tradition while rediscovering and repacka-
ging old proposals as though they were unprecedented, exciting, and modern; ignoring
or downgrading explicit criteria by which competing proposals can be evaluated."

Moden in der Wissenschaft äußern sich durch das exponentielle Wachstum von Subdisziplinen, Theorien, Paradigmen oder Methoden bezüglich ihrer Anhängerschaft bzw. der ihnen entgegengebrachten Aufmerksamkeit in der wissenschaftlichen Gemeinschaft. Aufgrund des kumulativen Charakters der wissenschaftlichen Wissensproduktion folgt auf den starken Anstieg an Aufmerksamkeit nicht unbedingt ein ebenso starker Rückgang der Aufmerksamkeit. Crane (1969a: 435-436) beobachtet für innovative Fachgebiete (,scientific fields“), die zur Modeerscheinung (,fashion") werden, eine S-förmige Kurve mit vier aufeinanderfolgenden Phasen: (1) langsames Wachstum, (2) exponentielles Wachstum, (3) langsames, lineares Wachstum und schließlich (4) langsamer Rückgang (ebd.). Eine solche Kurve ließe sich durch die Institutionalisierung der Mode erklären: Indem einschlägige Lehrstühle und Zeitschriften gegründet werden und indem Organisationen und persönliche Karrieren mit dem wissenschaftlichen Feld verknüpft werden, wird die Modeerscheinung zur Normalität. Sie hört also auf, Mode zu sein, auch wenn die Frequenz einschlägiger Veröffentlichungen auf deutlich höherem Niveau als vor der Mode stagniert (Bort und Kieser 2011: 673). 
Für die Mode bestimmter Konzepte, wie bspw. „organizational slack“ in der Organisationstheorie, gelangen Bort und Kieser (2011) hingegen zu einer glockenförmigen Kurve. Auf das Wachstum an Aufmerksamkeit folgt also ein ähnlich starker Rückgang, sodass ein neues Konzept in den Fokus gelangen kann. Verschiedene Erklärungen für diese Kurvenform sind möglich. So betont bspw. Starbuck (2009: 109): „These sequences of fads may have occurred in part because researchers value novelty and they have limited tolerance for imitation". Auch Generationenwechsel in der wissenschaftlichen Gemeinschaft, die gemäß Kuhn die Möglichkeit für junge Wissenschaftler*innen zur Etablierung eigener Ideen verbessern (Kuhn 1970 [1962]: 150-159), können Teil der Erklärung einer Glockenkurve der Aufmerksamkeit sein. Sperber (1990) betont außerdem den Einfluss wissenschaftsexterner Faktoren, die Wellen der Aufmerksamkeit innerhalb der Wissenschaft erzeugen können. So können bspw. Fördermittelgeber*innen ihre Zuwendungen in einem Forschungsbereich kurzfristig stark ausbauen und nach einem bestimmten Zeitraum wieder entziehen (Abrahamson 2009: 237). Dass der wachsende Einfluss der Medien zu einem „Hype Problem“ führt, analysiert insbesondere Weingart (2017), nach dem die Generierung öffentlicher Aufmerksamkeit zum Teilziel wissenschaftlicher Akteur*innen geworden ist, weshalb sich die wissenschaftliche Erkenntnisproduktion zunehmend an medialen Relevanzkriterien orientiert. Zudem kann gemäß Abrahamson (2009: 237) das Auf und Ab in der öffentlichen Sichtbarkeit eines sozialen Problems ein ebenso starkes Auf und $\mathrm{Ab}$ in der ihm entgegengebrachten wissenschaftlichen Aufmerksamkeit bewirken.

Auch „wissenschaftliche“ Gründe lassen sich anführen, wie Abrahamson (2009: 237) erklärt:

„It might be argued, for instance, that scientists jumped, in concert, to study so called ,cold fusion"-nuclear fusion occurring at near-ordinary temperatures and pressuresonly to abandon its study, in equal numbers, when the theory and evidence supporting the occurrence of cold fusion were refuted."

Kommt also eine Frage auf, deren Beantwortung als grundsätzlich für den Erkenntnisfortschritt einer ganzen Disziplin angesehen wird, mobilisiert die wissenschaftliche Gemeinschaft kurzfristig in großem Umfang Ressourcen zu ihrer Erforschung. Sobald die Frage als beantwortet gilt, werden diese Ressourcen wieder auf andere Fragen verwendet. Allerdings können Moden auch wiederkehren. So konstatiert Fleck bereits 1929 (S. 429): 
„Manche Gebiete der Naturwissenschaft liegen nach Jahren heftiger Entwicklung brach, wie z.t. heute die Anatomie, oder die zu Zeiten Keplers und Tycho Brahes so lebendige Astronomie. Sie scheinen fertig, tot. Aber eines Tages werden sie wieder lebendig, von anderem Standpunkt beleuchtet, mit neuen Begriffen wieder aufgenommen, durch пеие Bedürfnisse begehrenswert - und sind dann so frisch und, herrlich wie am ersten Tag“. “

Besonders häufig diskutierte Fragen im Zusammenhang mit Moden in der Wissenschaft sind, ob Moden einen positiven oder einen negativen Effekt auf die wissenschaftliche Entwicklung haben und ob Moden in der Wissenschaft vermeidbar sind (Bort und Kieser 2011: 672; Weingart 2017). Die Antwort hängt von dem jeweils vorliegenden Wissenschaftsverständnis $a b$, wobei sich insbesondere zwei opponierende Sichtweisen - eine rationalistische und eine pragmatische - voneinander abgrenzen lassen:

Auf der einen Seite werden Modeerscheinungen in der Wissenschaft häufig als ,the selection of problems on the basis of non-scientific criteria, as a form of deviance" dargestellt (Crane 1969a: 435). Abrahamson et al. (1989: 321) definieren sie als ,attractive concepts that fool scientists; a concept that receives more attention than it deserves “. Bei dieser Interpretation liegt ein rationalistisches Wissenschaftsbild vor, bei dem soziale Handlungsmotive wie die Hoffnung auf Fördermittel oder Anerkennung als ,nicht-wissenschaftlich“ eingestuft werden. Dieser Auslegung folgend sieht Hagstrom (1965: 177-194) in Modeerscheinungen ein motivationales Phänomen. Wissenschaftler*innen, die Moden folgen, wählen ihre Forschungsgegenstände demnach nicht auf Grundlage eigener, theoretischer Erwägungen, sondern weil sie sich dadurch Anerkennung in ihrer Fachgemeinschaft erhoffen. Das eigene Urteil gerate dabei gegenüber dem ihrer Fachkolleg*innen in den Hintergrund (Crane 1969a: 435). Moden seien daher schädlich für die Entwicklung der Wissenschaft, weil Ressourcen von ,signifikanten “ Forschungsfeldern zugunsten ,,weniger wichtiger“ Felder abgezogen würden (ebd.). Das Ausmaß, in dem Modeerscheinungen auftreten, weise auf die Ineffizienz der wissenschaftlichen Gemeinschaft hin sowie auf ein ,public support system that is not getting the most for its dollars" (Abrahamson et al. 1989: 321).

Auch Starbuck (2009: 242) stuft Modeerscheinungen als schädlich für die Wissenschaft ein:

„Faddishness in social and behavioral research is troublesome partly because it involves the endless rediscovery of what could have been accumulated as knowledge. Faddishness also wastes research resources by encouraging imitation, and it corrupts evaluation of research outcomes by fostering the naive appraisal that if many people 
are enthusiastically taking similar actions, those actions must be worthwhile. Unfortunately, research themes and methods that advocates have proudly claimed to be new and better have had a history of rediscovering and renaming hoary commonplaces. "

Als ursächlich für die ",faddishness “ der Wissenschaft sieht Starbuck neben anderen Faktoren die Massenproduktion wissenschaftlicher Studien, die dazu führe, dass zu viel , unnütze Spreu “ (, useless chaff") in Fachzeitschriften veröffentlicht werde (Starbuck 2009: 109). Um gegen solche Modeerscheinungen vorzugehen, seien Indikatoren zu entwickeln und flächendeckend anzuwenden, anhand derer die Effektivität der Forschung gemessen werden könne (ebd.: 114). Allerdings lassen die Darstellungen Starbucks einige bislang ungeklärte Fragen aufkommen: ,Wie lässt sich die Effektivität der Forschung messen?' (vgl. Kantelinen 2009: 230), , Wie lässt sich ein Konsens darüber etablieren, was gute und effektive Forschung ist und wie sie von unnützer Mode zu unterscheiden ist?' Und letztlich: ,Ist die Beurteilung, was gute und effektive Forschung konstituiert, nicht ebenfalls von Moden beeinflusst?' (vgl. Abrahamson 2009: 236).

In Opposition zu der dargestellten Sichtweise, Moden seien schädlich und die Wissenschaft vor ihnen zu verteidigen, plädiert eine Reihe von Autor*innen für einen pragmatischen Umgang mit Moden in der Wissenschaft. Sie halten Modeerscheinungen nicht nur für unvermeidbar, sondern verbinden auch einige Vorteile mit ihnen. So würden Moden das starre Festhalten an alten Theorien verhindern und stattdessen Raum für Innovation schaffen (Abrahamson 2009: 238). Wenn Wissenschaftler*innen eine Mode adaptieren, passen sie diese für ihren eigenen Gebrauch an und übernehmen im besten Fall nur diejenigen Komponenten der Mode, die sie im Kontext ihrer jeweiligen Forschung für geeignet erachten, während sie solche außen vor lassen, die ihren bisher verwendeten Ansätzen unterlegen zu sein scheinen. Durch diese Übersetzung und das Übernehmen potenziell überlegener Theoriebestandteile verändere sich nicht nur die Forschung der einzelnen Person, sondern auch der Gegenstand der Mode selbst, was - so die Annahme - einen epistemischen Mehrwert verspreche (Czarniawska 2008: 851; Bort und Kieser 2011: 656). Außerdem biete eine Mode Ordnung und Orientierung in der kollektiven Wissensproduktion und gewährleiste, dass aus dem überwältigenden Angebot aller verfügbaren Ansätze eine Auswahl als bekannt und mehr oder weniger gültig vorausgesetzt werden könne (Blumer 1969: 289). Die Mode schaffe daher eine temporäre Kohäsion der Fachgemeinschaft, im Rahmen derer neue Austauschkanäle geschaffen würden, sodass verschiedene Subgemeinschaften - vermittelt über eine gemeinsame Mode - vorübergehend in den Austausch treten könnten. 
Sherman (1996) teilt die Ansicht, dass Modeerscheinungen in der Wissenschaft nicht vermeidbar sind und stellt die Schaffung eines Bewusstseins darüber in den Vordergrund. Er kommt zu dem Schluss, dass Wissenschaftler*innen die Moden - und damit die eigene Disziplin - stärker selbst gestalten können, wenn sie die Existenz des ,fashion process " anerkennen:

\begin{abstract}
„The operation of the fashion process in a discipline may either allow - or indeed encourage - the adoption of radical new approaches, or it may provide the appearance of change through a continuous reshuffle of existing fashions while minimizing true change. The process can be used as a funnel, a sledgehammer, a door, or a window. The recognition of the fashion process by a disciplinary mainstream, however, does provide a mechanism to maximize the potential benefits by increasing the participation in decision-making. " (Sherman 1996: 98)
\end{abstract}

\title{
Schlussfolgerungen
}

Wie in dem Streit zwischen Popper und Kuhn (siehe Kp. 1.3.1), besteht in den genannten Ansätzen Einigkeit darüber, dass Moden sich nicht im Rahmen eines rationalistischen Wissenschaftsverständnisses erklären lassen, sondern dass soziale Faktoren - anstelle von theoretischen oder logischen - ausschlaggebend für deren Entwicklung sein müssen. Der grundlegende Unterschied zwischen den Sichtweisen liegt in der Frage, ob dieses Abweichen von der rationalen Erkenntnisentstehung als ein zu beseitigender Schwachpunkt des Wissenschaftssystems interpretiert wird, oder als der nicht vermeidbare und nicht unbedingt schädliche Normalzustand wissenschaftlicher Erkenntnisentstehung. Die Stärken dieser nur teilweise aufeinander Bezug nehmenden Sammlung von Ansätzen liegen weniger in der Beschreibung und Erklärung wissenschaftlicher Transformationen, sondern vielmehr in ihrer Bewertung. Durch das Aufzeigen von Faktoren und Mechanismen einer wissenschaftlichen Mode legen beide Fraktionen - die Rationalist*innen und die Pragmatiker*innen - jeweils überzeugende Argumente vor, die im Rahmen der empirischen Betrachtung der Themenkarrieren sowie in der abschließenden Diskussion dieser Arbeit aufzugreifen sind. Ein weiterer Vorzug liegt darin, dass - fraktionsübergreifend - ein Bewusstsein von und ein offener Umgang mit Modeerscheinungen angemahnt wird. Schließlich können die Prozesse und Effekte einer Modeerscheinung nur dann kontrolliert, gesteuert und genutzt werden, wenn ihre Existenz anerkannt wird.

In dieser Arbeit wird der Begriff der Mode aus zwei Gründen für die Bezeichnung des untersuchten Phänomens vermieden. Zum einen ist in dem Begriff neben einer breiten Diffusion des Gegenstands, auch dessen prinzipielle Vergänglichkeit 
angelegt (Zapf 1989: 177). Für die im Rahmen dieser Arbeit untersuchten Themen in der Planungswissenschaft ist allerdings eher eine Institutionalisierung als ein Verschwinden auszumachen. Zum anderen erzeugt der Begriff automatisch eine Dichotomie, bei der eine, rationale Wissenschaft' abseits von Moden nahegelegt wird, die von sozialen und ästhetischen Kriterien der Erkenntnisproduktion unabhängig zu sein scheint. Damit einhergehend werden Moden tendenziell als negativ und , unwissenschaftlich ' abgewertet, wie zahlreiche Veröffentlichungen belegen. Diese Abwertung von Themenkarrieren ist nicht das Ziel dieser Arbeit, wohl aber das Verstehen ihrer Mechanismen und das Schaffen eines Bewusstseins für deren Existenz.

Open Access Dieses Kapitel wird unter der Creative Commons Namensnennung 4.0 International Lizenz (http://creativecommons.org/licenses/by/4.0/deed.de) veröffentlicht, welche die Nutzung, Vervielfältigung, Bearbeitung, Verbreitung und Wiedergabe in jeglichem Medium und Format erlaubt, sofern Sie den/die ursprünglichen Autor(en) und die Quelle ordnungsgemäß nennen, einen Link zur Creative Commons Lizenz beifügen und angeben, ob Änderungen vorgenommen wurden.

Die in diesem Kapitel enthaltenen Bilder und sonstiges Drittmaterial unterliegen ebenfalls der genannten Creative Commons Lizenz, sofern sich aus der Abbildungslegende nichts anderes ergibt. Sofern das betreffende Material nicht unter der genannten Creative Commons Lizenz steht und die betreffende Handlung nicht nach gesetzlichen Vorschriften erlaubt ist, ist für die oben aufgeführten Weiterverwendungen des Materials die Einwilligung des jeweiligen Rechteinhabers einzuholen.

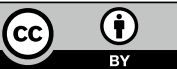

